\title{
Non-Coronary Cusp of the Aortic Valve
}

National Cancer Institute

\section{Source}

National Cancer Institute. Non-Coronary Cusp of the Aortic Valve. NCI Thesaurus. Code C127639.

The cusp of the aortic valve that is positioned posteriorly relative to the other two aortic cusps. 